\title{
Identification of a novel variant of the ciliopathic gene FUZZY associated with craniosynostosis
}

\author{
William B. Barrell (D) ${ }^{1}$, Hadeel Adel Al-Lami (D) ${ }^{1,2}$, Jacqueline A. C. Goos ${ }^{3}$, Sigrid M. A. Swagemakers ${ }^{4}$, Marieke van Dooren ${ }^{3,5}$, \\ Elena Torban $\mathbb{D}^{6}$, Peter J. van der Spek $\mathbb{D}^{4}$, Irene M. J. Mathijssen ${ }^{3}$ and Karen J. Liu $\mathbb{D}^{1 凶}$
}

(c) The Author(s) 2021

\begin{abstract}
Craniosynostosis is a birth defect occurring in approximately one in 2000 live births, where premature fusion of the cranial bones inhibits growth of the skull during critical periods of brain development. The resulting changes in skull shape can lead to compression of the brain, causing severe complications. While we have some understanding of the molecular pathology of craniosynostosis, a large proportion of cases are of unknown genetic aetiology. Based on studies in mouse, we previously proposed that the ciliopathy gene Fuz should be considered a candidate craniosynostosis gene. Here, we report a novel variant of FUZ (c.851 G > C, p.(Arg284Pro)) found in monozygotic twins presenting with craniosynostosis. To investigate whether Fuz has a direct role in regulating osteogenic fate and mineralisation, we cultured primary osteoblasts and mouse embryonic fibroblasts (MEFs) from Fuz mutant mice. Loss of Fuz resulted in increased osteoblastic mineralisation. This suggests that FUZ protein normally acts as a negative regulator of osteogenesis. We then used Fuz mutant MEFs, which lose functional primary cilia, to test whether the FUZ p.(Arg284Pro) variant could restore FUZ function during ciliogenesis. We found that expression of the FUZ p.(Arg284Pro) variant was sufficient to partially restore cilia numbers, but did not mediate a comparable response to Hedgehog pathway activation. Together, this suggests the osteogenic effects of FUZ p.(Arg284Pro) do not depend upon initiation of ciliogenesis.
\end{abstract}

European Journal of Human Genetics (2022) 30:282-290; https://doi.org/10.1038/s41431-021-00988-6

\section{INTRODUCTION}

Craniosynostosis is the premature fusion of one or more sutures of the skull vault and has an incidence of (approximately) 1:2000 live births [1-3]. While there are syndromes that present with craniosynostosis (sometimes with known genetic aetiology), the genetic associations of the more common non-syndromic craniosynostosis (approximately 85\%) is less well understood [4]. Craniosynostosis is closely linked to the development of the cranial bones and sutures. During embryogenesis, there are condensations of neural crest (broadly anterior) and mesodermally (broadly posterior) derived skeletal progenitors. These cells then proliferate and differentiate into osteoblasts that go on to lay down matrix (osteoid). This matrix is then mineralised to form bone. Between the growth front of these bones the mesenchymal sutures work to prevent premature fusion. Pathological fusion can result from aberrant specification of the sutural mesenchyme towards the bone lineage, increased osteoblastic/decreased osteoclastic activity, or from aberrant mechanical forces causing bone fusion at close approximating surfaces [5]. Craniosynostosis can lead to raised intracranial pressure, which can impair eyesight and mental development $[1,6,7]$. There are good surgical treatment options for craniosynostosis; however, genetic sequencing is crucial for our understanding of the developmental aetiology of this disorder.
Here, we report a novel variant in the human FUZZY gene as a candidate for craniosynostosis, identified from whole genome sequencing (WGS) of a pair of monozygotic twins with craniosynostosis (case 1 and 2, Table 1, Fig. 1A) and their consanguineous clinically unaffected parents. Just over a handful of pathological variants of FUZ have previously been identified (cases 3-6, Table 1); three cases with severe neural tube defects (cases 3-5, Table 1) [8] and one case with the embryonically lethal, short-rib polydactyly syndrome II-like phenotype (case 6, Table 1) [9]. In addition, Zhang et al. 2018 reports the identification of FUZ p.(Arg284Leu), described as an "unsolved case" diagnosed as asphyxiating thoracic dystrophy (ATD) with polydactyly (case 7, Table 1). The fact that so few variants are present in the literature suggests that full loss-of-function changes are lethal, and case 6 further supports this as this early truncation is likely loss-offunction.

The human FUZ protein is comprised of 418 amino acids and shares $86.364 \%$ sequence homology with mouse FUZ. A number of key protein-protein interactions have been identified, although no specific binding sites have been established (Fig. 1D). FUZ has 3 putative longin domains (Fig. $1 \mathrm{E}$ ), which function in membrane and vesicular trafficking [10-15]. The FUZ gene was first identified as a planar cell polarity (PCP) effector in Drosophila $[16,17]$. More recent research from our lab and others has highlighted the role

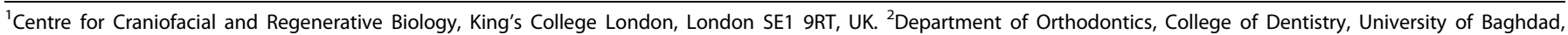

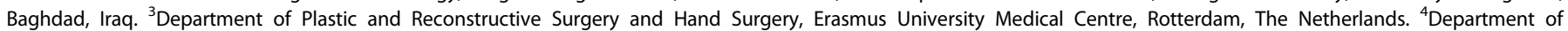

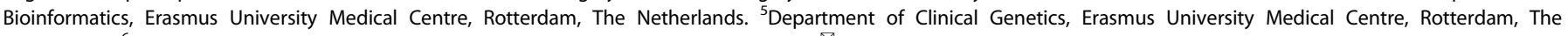
Netherlands. ${ }^{6}$ Department of Medicine, McGill University Health Centre, Montreal, Canada. ${ }^{\bowtie}$ email: karen.liu@kcl.ac.uk 
of Fuz in intraflagellar transport within the primary cilium, a cellular organelle involved in cell-cell signalling, mechanosensation and signal transduction. Animal models have implicated Fuz in Hedgehog (Hh), fibroblast growth factor (FGF) and Wnt signalling pathways; $[12,13,18,19]$ these pathways have all been associated with congenital craniofacial anomalies.

Primary cilia develop in the process of ciliogenesis, where centrioles form basal bodies and dock with the cortex. The microtubules in the cilium are then nucleated from the basal body. Because there is no protein synthesis in the cilium, proteins necessary for cilium function must be actively trafficked to the cilium. Within the cilium this is achieved via the intraflagellar transport (IFT) mechanism [20]. Functional cilia and IFT have both been shown to be crucial for Hh signal transduction [21].

In the absence of $\mathrm{Hh}$ ligand, the transmembrane receptor Smoothened (SMO) is inhibited by a second receptor, Patched (PTCH). In this context, downstream GLI transcription factors are processed to repressor forms. Upon ligand binding to PTCH, SMO is activated, allowing relocalisation of the GLI proteins to the cilium. This translocation via the IFT machinery is necessary for processing of GLI2 into a transcriptional activator. A number of craniofacial anomalies have been associated with cilia, IFT and Hedgehog signalling, including holoprosencephaly, cranioectodermal dysplasia, and ATD, mentioned above $[22,23]$. In mouse models, mutation in cilia genes, IFT and Hedgehog pathway genes all have severe craniofacial phenotypes, frequently precluding study of the calvaria. Therefore, perturbations of primary cilium associated proteins have not been directly correlated with craniosynostosis [22-24]. However, Indian Hedgehog mutants show reduced ossification in the skull [25] and a role in endochondral ossification [26, 27].

In the context of vesicular trafficking, FUZ physically interacts with the small GTPase RSG1 [12, 13], and indeed a mouse knockout of Rsg1 mostly phenocopies Fuz knockouts [28]. During ciliogenesis, FUZ is known to interact with Inturned and WDPCP (in the ciliogenesis and planar polarity effector (CPLANE) complex) and the IFT-A subunits, which are involved in retrograde intraflagellar transport in the primary cilium [13, 19]. Specifically, IFT-A particles were not localised to the basal body in fuz morphant frog embryos. Finally, the CPLANE complex also interacts with chaperonin/CCT complex [13]. This is similar to the BBS6, 10 and 12 complex that helps form the BBSome, another crucial protein complex for primary cilia function. These chaperonin complexes do not enter the cilium but are involved in complex assembly necessary for primary cilium function. Overall, it is clear that FUZ is crucial for the transport of components to the primary cilium and potentially promotes protein complex assembly necessary for downstream cilium formation and function.

Recent work has shown that Fuz depletion leads to a gradual loss of the cilium, due to a failure of the retrograde intraflagellar transport machinery [19]. This explains why Fuz loss-of-function mouse mutants do not demonstrate early embryonic lethality seen in core ciliogenesis or IFT pathway genes. Nevertheless, we have shown that Fuz is required in the cranial neural crest, where Fuz mutation leads to a failure to process Gli3 repressors, which was phenocopied in the Gli3 mouse mutants [29]. This resulted in de-repression of fibroblast growth factor-8 (FGF-8) expression in the head and ectopic expansion of the neural crest domain [29]. This led us to the conclusion that craniosynostosis seen in our mouse models was mimicking that seen in syndromic craniosynostosis models, namely Crouzon and Apert Syndromes, which result from genetic increases in FGF signalling.

Our previous studies demonstrated that complete knockout of Fuz in a mouse model leads to prenatal lethality, coronal craniosynostosis, micrognathia, facial malformations, eye, and heart defects [12, 24, 29-32]. In this mouse model, we identified several key roles for Fuz in skull development: first, mutation of Fuz results in an expansion of cranial neural crest, leading to an 


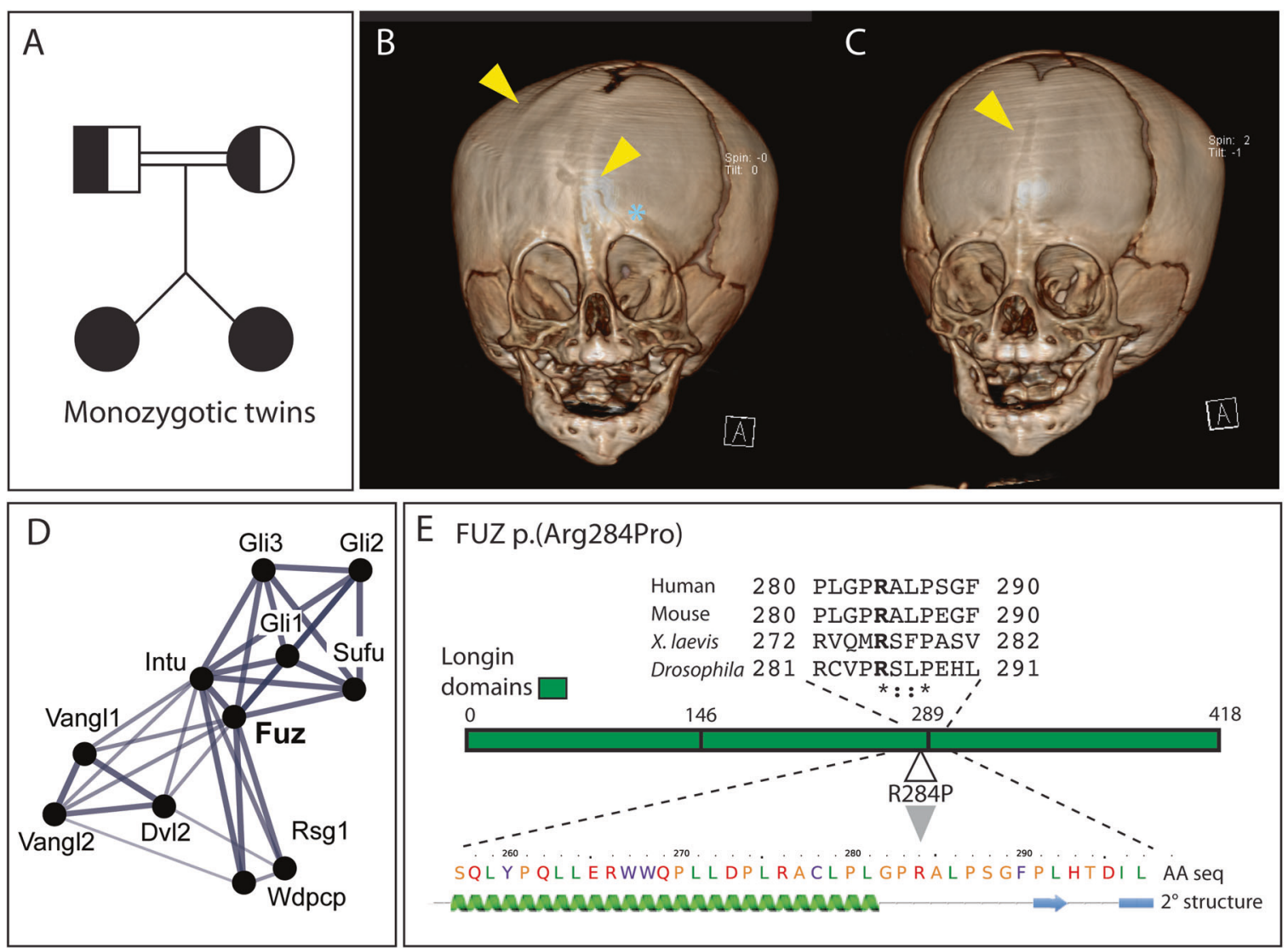

Fig. 1 Novel missense variant: FUZ p.(Arg284Pro). Family pedigree scheme (A). Computed tomography (CT) scan reconstructions of twins exhibiting metopic craniosynostosis (B and C, yellow arrowhead, midline). The twin in (B) also exhibits unilateral coronal craniosynostosis (B, yellow arrowhead, lateral). Note the resultant dysmorphism due to synostosis (B, blue asterisk). D STRING network summary depiction of known and predicted FUZ protein interactions. Line thickness denotes confidence levels, with thick lines showing highest confidence (0.9). E FUZ protein scheme with alignment of relevant Human, Mouse, Xenopus laevis and Drosophila regions. Predicted longin domains are shown $(E, *$ denotes conserved residues,: denotes residues with strongly similar groups and. denotes residues with weakly similar groups). PHYRE2 secondary structure modelling ( $E$, green spiral $=\alpha$ helix, blue arrow $=\beta$ sheet).

Table 2. Homozygous variants found through WGS not present in the wellderly cohort.

\begin{tabular}{|c|c|c|c|c|c|c|c|}
\hline Chrom & Position (hg19) & Ref base & Alt base & Gene & Transcript & DNA change & Protein change \\
\hline chr1 & 43770666 & G & A & TIE1 & ENST00000372476.8 & c. $203 \mathrm{G}>\mathrm{A}$ & p.(Arg68His) \\
\hline chr9 & 90503521 & A & C & SPATA31E1 & ENST00000325643.6 & c. $4119 \mathrm{~A}>\mathrm{C}$ & p.(Arg1373Ser) \\
\hline chr9 & 95142080 & G & A & CENPP & ENST00000375587.8 & c. $503 \mathrm{G}>\mathrm{A}$ & p.(Arg168Gln) \\
\hline chr9 & 96439169 & G & C & PHF2 & ENST00000359246.9 & c. $3126 \mathrm{G}>\mathrm{C}$ & p.(Gln1042His) \\
\hline chr10 & 64913893 & A & G & NRBF2 & ENST00000277746.11 & c. $779 \mathrm{~A}>\mathrm{G}$ & p.(Asn260Ser) \\
\hline chr10 & 69991419 & G & $\mathrm{T}$ & ATOH7 & ENST00000373673.5 & c. $16 \mathrm{C}>\mathrm{A}$ & p.(Pro6Thr) \\
\hline chr13 & 42623060 & C & A & $D G K H$ & ENST00000337343.8 & c. 151 C > A & p.(Leu51Met) \\
\hline chr14 & 105415433 & T & C & AHNAK2 & ENST00000333244.6 & c. 6355 A > G & p.(Met2119Val) \\
\hline chr19 & 50312016 & $\mathrm{C}$ & G & FUZ & ENST00000313777.9 & $\mathrm{c} .851 \mathrm{G}>\mathrm{C}$ & p.(Arg284Pro) \\
\hline chr19 & 55236006 & C & $\mathrm{T}$ & KIR3DL3 & ENST00000291860.1 & c. $5 \mathrm{C}>\mathrm{T}$ & p.(Ser2Leu) \\
\hline
\end{tabular}

increase in skeletogenic precursors in the head [29]. Second, using an Osx-1::GFP-cre reporter line to lineage label the osteoblast precursors, we showed that the frontal bone mesenchyme was expanded at the expense of the parietal bone [30], which could manifest as craniosynostosis, or an absence of the coronal suture in late gestation animals. These studies identified a clear role for FUZ in early establishment of cell fate and cranial bone tissue boundaries. While mouse Fuz is expressed throughout craniofacial structures [31], FUZ itself had not previously been directly implicated in osteoblast-specific development or bone formation.

In this study, we report the first craniosynostosis associated variant of FUZ and propose a novel function of FUZ during the later stages of cranial bone development, using a mouse model to demonstrate that loss of FUZ leads to excessive ossification. Furthermore, we find that the novel FUZ p.(Arg284Pro) variant can partially rescue ciliogenesis function in Fuz mutant mouse 
Table 3. Clinical observations and phenotypes.

\begin{tabular}{|c|c|c|}
\hline & Case 1 & Case 2 \\
\hline Craniosynostosis & Metopic and coronal suture synostosis & Metopic suture synostosis \\
\hline \multirow[t]{4}{*}{ Eye } & $\begin{array}{l}\text { Fundoscopy: papilledema OS > OD, no Drusen, no } \\
\text { ophthalmologic cause }\end{array}$ & Fundoscopy: papilledema both eyes \\
\hline & Skiascopy right eye: $\mathrm{S}+3.25=\mathrm{C}-1.0 \mathrm{AS} 90$; & Skiaskopy right eye: $\mathrm{S}+2.50=\mathrm{C}-0.50 \mathrm{AS} 6$; \\
\hline & Left eye: $S+6.50=C-1.0$ AS 180 & Left eye: $S+2.50=C-0.50$ AS 154 \\
\hline & Ishihara OD $0.5 / 8 \mathrm{ft}$ OS $0.5 / 8 \mathrm{ft}$ & Wears glasses \\
\hline \multirow[t]{2}{*}{ Ear } & Prominent ears & Prominent ears \\
\hline & Right ear larger than left & Frequent ear infections; tubes placed 3 times \\
\hline Endocrine & $\mathrm{N} / \mathrm{A}$ & Premature puberty, started hormonal therapy \\
\hline \multirow[t]{2}{*}{ Skull morphometrics notes } & $\begin{array}{l}\text { Skull circumference: initially }-1 \text {, moving to }-1.5 \text { SD since age } \\
5 \text { years }\end{array}$ & $\begin{array}{l}\text { Skull circumference: }-1 S D \text {, moving to }-1.5 \text { SD } \\
\text { since age } 7 \text { years }\end{array}$ \\
\hline & Skull length: $-3 S D$ & Skull length: $-2.5 \mathrm{SD}$ \\
\hline \multirow[t]{7}{*}{ Other observations } & Developmental delay & Developmental delay \\
\hline & Low Hairline & Low hairline \\
\hline & Narrow nose & Hypopigmentation caudally of the left nipple \\
\hline & Ultrasound abdomen aged 6 years: normal & Ultrasound of abdomen aged 6 years: normal \\
\hline & $\begin{array}{l}\text { CT and MRI aged } 6 \text { months and MRI aged } 18 \text { months: agenesis } \\
\text { of the callosal body, stable wide lateral and third ventricles }\end{array}$ & $\begin{array}{l}\text { CT scan aged } 6 \text { months: stable dilatation of lateral } \\
\text { ventricles }\end{array}$ \\
\hline & MRI scan aged $81 / 2$ years: unchanged dilatation of ventricles & \multirow{2}{*}{$\begin{array}{l}\text { MRI scan aged } 8 \frac{1}{2} \text { years: dilatation of ventricles, } \\
\text { mild agenesis of the callosal body }\end{array}$} \\
\hline & $\begin{array}{l}\text { Increased intracranial pressure requiring occipital expansion } \\
\text { aged } 8 \text { years }\end{array}$ & \\
\hline Birth observations & $\begin{array}{l}\text { At birth } 1870 \text { gram, skull circumference } 31.5 \mathrm{~cm} \text {, gestation } \\
36 \text { weeks and } 4 \text { days }\end{array}$ & $\begin{array}{l}\text { At birth } 2125 \text { grams, skull circumference } 31 \text { cm, } \\
\text { gestation } 36 \text { weeks and } 4 \text { days }\end{array}$ \\
\hline
\end{tabular}

embryonic fibroblasts, suggesting that this variant may reveal cilia dependent and independent functions of FUZ.

\section{RESULTS}

Novel missense mutation in FUZ identified in twins presenting with craniosynostosis

We performed whole genome sequencing and identified a novel homozygous variant in FUZ (c.851 G > C, p.(Arg284Pro)) (Table 1, Fig. 1) in monozygotic twinned, female infants presenting with craniosynostosis. The consanguineous parents (paternal grandfather and maternal grandmother are siblings) are both heterozygous carriers, suggesting that this variant is recessive. Other homozygous variants found that are not present in the control population are presented in Table 2 (12 variants total). SIFT and PolyPhen scores suggested that these other variants were not pathogenic (Supplemental Table 1). The only variant predicted to be involved in craniofacial osteogenesis was FUZ. In addition, this novel mutation has not been reported in allele frequency reference databases such as (GnomAD) but is in the same position as an "unsolved case" (p.(Arg284Leu), case 7, Table 1). Moreover, the calculated SIFT and PolyPhen scores were indicative for a pathogenic variant (Supplemental Table 1).

Both patients presented at 6 months old with metopic suture craniosynostosis (Fig. 1B-C, yellow arrowhead). Additionally, case 1 (Table 1) presented with unilateral right coronal synostosis (Fig. 1B, yellow arrowhead). While the metopic suture usually fuses between 3-9 months, the coronal suture should remain patent until 20+ years [1]. Both patients also had dilatation of the lateral brain ventricles, with agenesis of the corpus callosum present in case 1 (data not shown). The twins were not reported to have any other phenotypes associated with FUZ knockout models, suggesting that this allele may not be a complete loss of function. Further relevant clinical observations are presented in Table 3.

Key protein-protein interactions of FUZ via STRING analysis indicate roles in PCP signalling (Vangl1, Vangl2, Dvl2 and Intu),
Hedgehog signalling (Gli1, Gli2, Gli3 and Sufu) and in the CPLANE complex (Intu, Rsg1 and Wdpcp) (Fig. 1D). Within FUZ, the arginine residue at amino acid position 284 is conserved across human, Xenopus, mouse, and Drosophila and lies adjacent to the C-terminal longin domain (Fig. 1E), which is thought to mediate vesicular trafficking [10-12]. This mutation is predicted to be deleterious (CADD score 25.4 (CADD Exome (1.6.1)). Arg284 lies outside of the predicted a helices or $\beta$ sheets in FUZ (Fig. 1, E) suggesting that any structural change from the Arg284Pro variant is likely to be in the tertiary structure of the protein rather than affecting secondary structure. Other reported FUZ gene variants (Table 1) are either missense or truncating and will be discussed further in discussion. However, none are associated with craniosynostosis.

\section{The role of FUZ during craniofacial ossification}

To date most research has focused on the requirements of FUZ during neural crest induction, and toward implications for patterning of the craniofacial skeleton. Specific roles during osteogenesis or bone formation have not been explored. Given the craniosynostosis observed in these patients, we set out to test whether mouse Fuz is required during craniofacial osteoblast mineralisation. Homozygous Fuz mutant mice exhibit $100 \%$ prenatal lethality with a progressive loss from embryonic day 13.5 (E13.5) onwards. Therefore heterozygous $\mathrm{Fuz}^{+/-}$mice were intercrossed to generate control wild-type, heterozygous and homozygous null animals, which were collected at embryonic day 18 (E18.5), just prior to birth. Primary osteoblasts were isolated from the skull vaults and mandibles for culturing in a mineralisation assay (Fig. 2). Pre-osteoblasts were assessed based on alkaline phosphatase levels (red staining), while von Kossa staining was used to determine overall mineralisation levels (black staining). Interestingly, Fuz mutant osteoblasts from both skull vault and mandible showed increased mineralisation compared to the wildtype and heterozygous controls (Fig. 2, black staining in $\mathrm{D}, \mathrm{E}, \mathrm{I}$ and $\mathrm{J}$ compared to $\mathrm{A}-\mathrm{C}$ and $\mathrm{F}-\mathrm{H}$ ). 


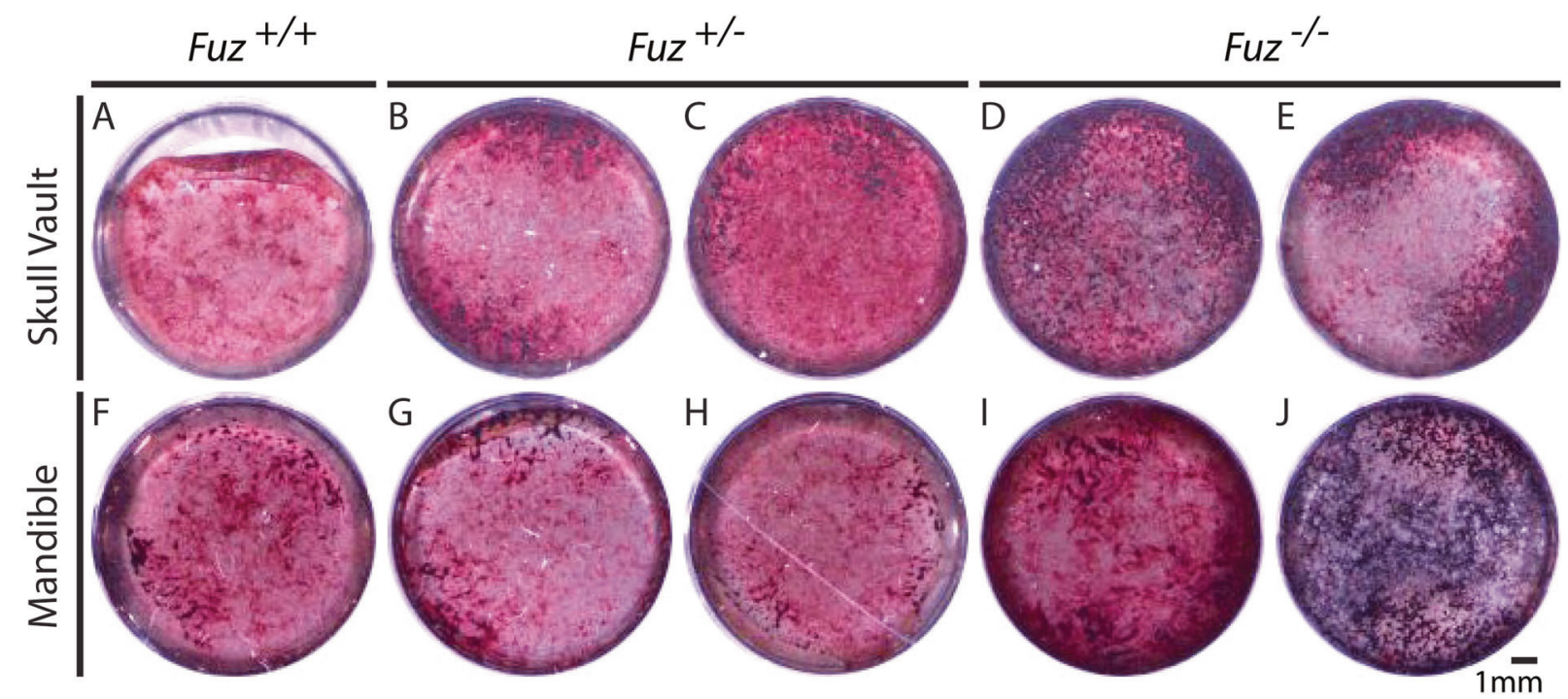

Day 7
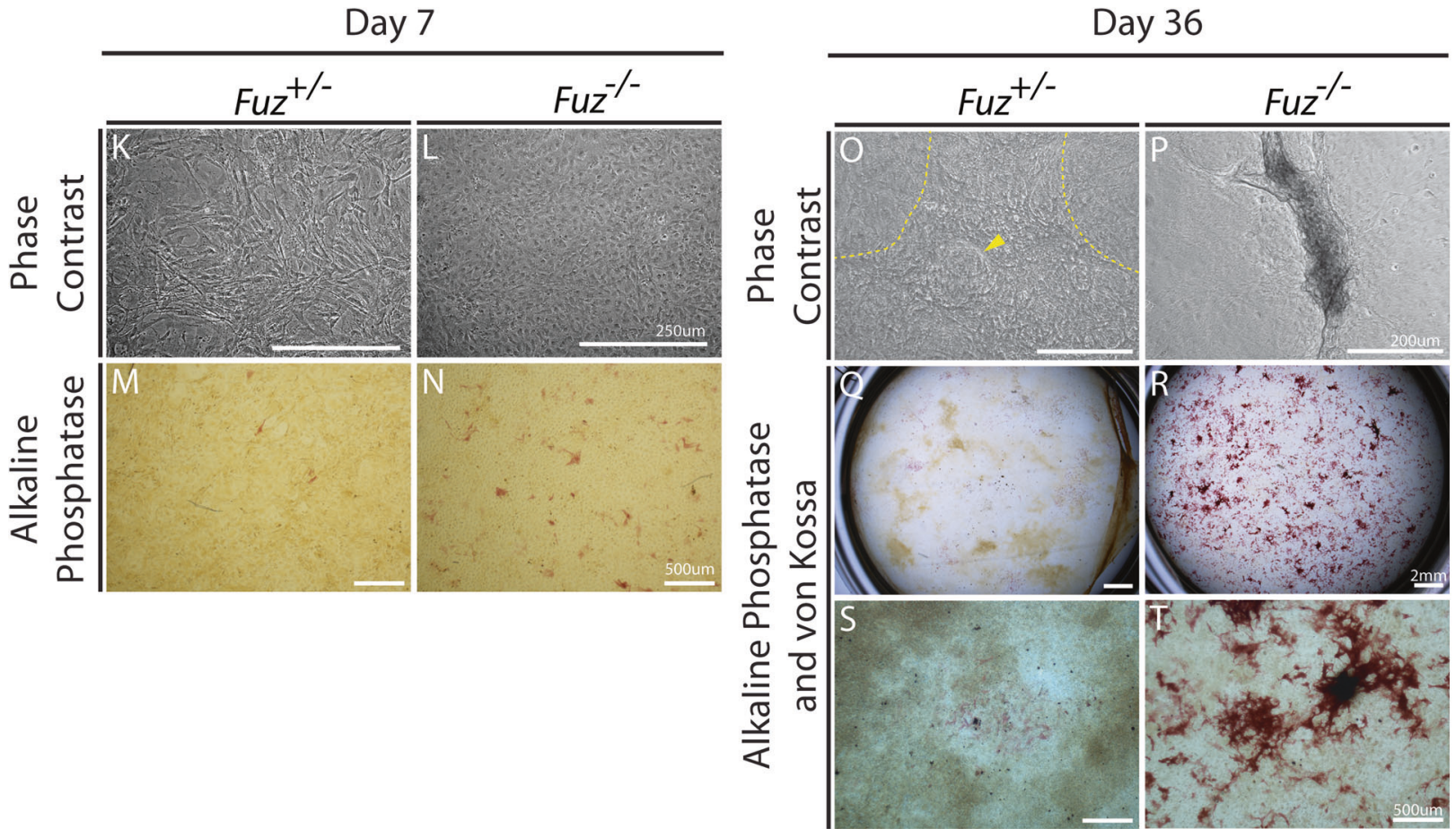

Fig. 2 Fuz mutant cells exhibit increased mineralisation in vitro. A-J Skull vault and mandibular bones were dissected from E18.5 foetuses. Isolated osteoblasts were cultured for 14 days and assayed for alkaline phosphatase activity (red) and mineral deposition (black, Von Kossa staining). Increased mineralisation was seen in Fuz ${ }^{-1-}$ cultures (skull vault; $\mathbf{D}, \mathbf{E}$, mandible; I, J) compared to controls (Fuz ${ }^{+/+}$(Skull vault; A, Mandible; F) and Fuz ${ }^{+-}$(Skull vault; B, C, Mandible; G, H). K-T Primary mouse embryonic fibroblasts were isolated from E12.5 animals and cultured in osteogenic media for seven days $(\mathbf{K}-\mathbf{N})$ or 36 days $(\mathbf{O}-\mathbf{P})$. At seven days, controls were fibroblastic (K) while mutant cells showed a cobblestoned appearance (L) with increased alkaline phosphatase expression ( $\mathbf{N}$ ). By day 36 , cellular condensations were seen in controls $(\mathbf{O}$, yellow arrowhead and dashed lines) whereas mutants exhibited refractive, mineralised nodules (P). No nodules were seen in control cultures. Mutant cultures had vastly increased alkaline phosphatase expression compared with controls (compare $\mathbf{R} \& \mathbf{T}$ to $\mathbf{Q} \& \mathbf{S}$ ). Mutants showed some alkaline phosphatase positive nodules (red) co-staining with Von Kossa stain (black) (compare $\mathbf{T}$ to $\mathbf{S}$ ). Scale bars as indicated.

This suggests that the mineralisation potential of Fuz mutant osteoblasts is increased.

Because the severe prenatal lethality seen in the $\mathrm{Fuz}^{-/-}$mice limited our access to calvarial osteoblasts, we then turned to mouse embryonic fibroblasts (MEFs) to further confirm the proosteogenic phenotypes. MEFs can be induced to undergo osteogenesis when challenged with media containing bone morphogenetic protein-2 (BMP-2). Within 7 days, Fuz mutant cells had a more cobblestoned appearance compared to the more fibroblastic wildtype cells (Fig. 2K, L). In addition, an increase in the area of alkaline phosphatase positive cells was seen (red staining Fig. $2 \mathrm{M}$ and $\mathrm{N}$ ). By 36 days in culture, the appearance of the cultures was very different, with Fuz mutant cells showing many refractive, mineralised alkaline phosphatase positive nodules (Fig. 2O-T).

\section{FUZ p.(Arg284Pro) can partially rescue FUZ mutant ciliogenesis}

If the function of FUZ is impaired by the p.(Arg284Pro) variant, then we might expect to see that protein localisation, ciliogenesis, 


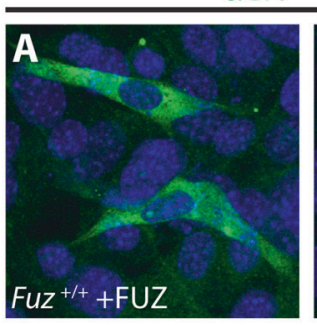

Hoescht
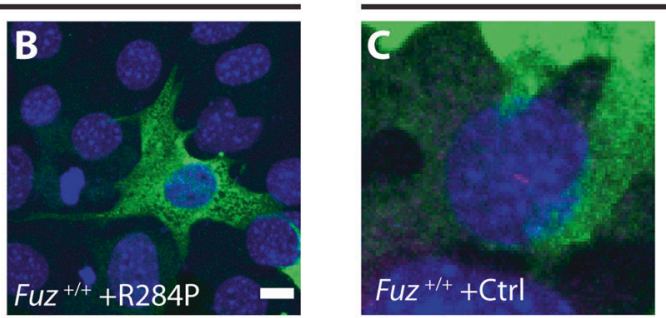

aGFP $\alpha$ Arl13b Hoescht

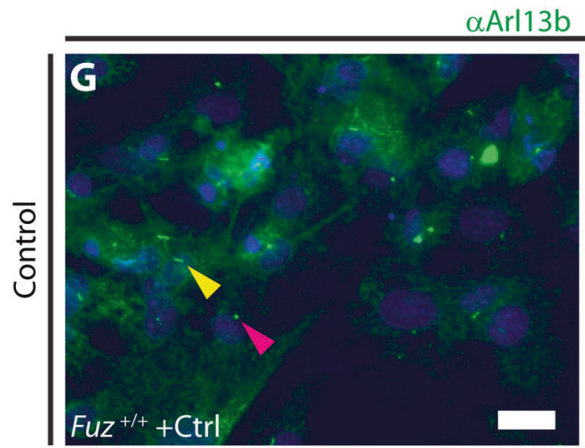

Hoescht
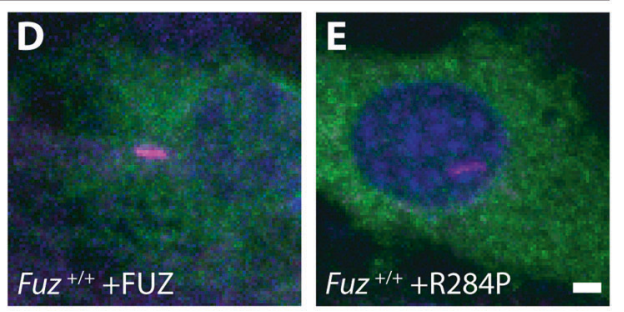

$\mathbf{F}$

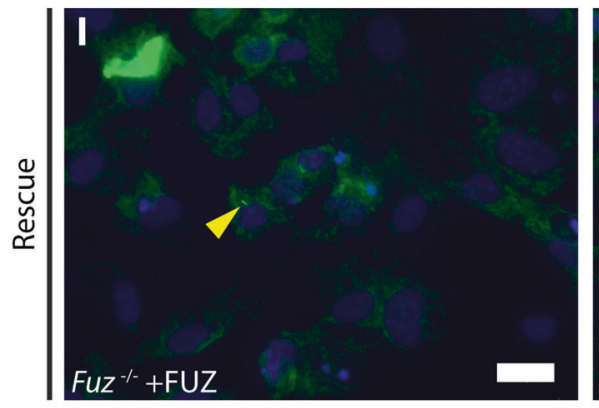

$\mathbf{L}$

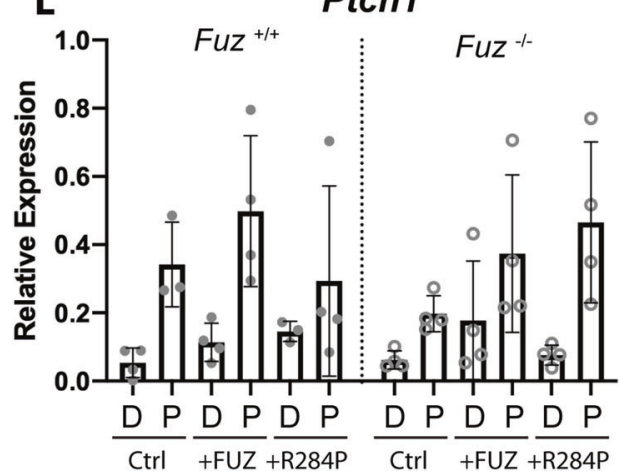

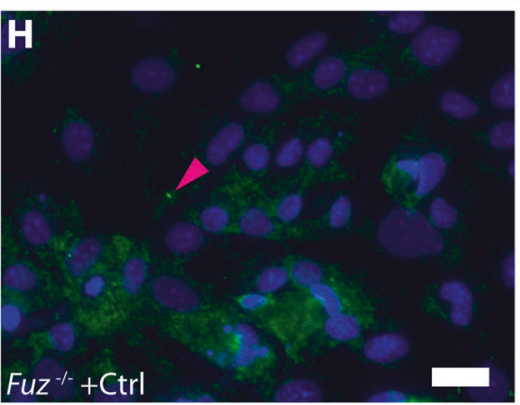

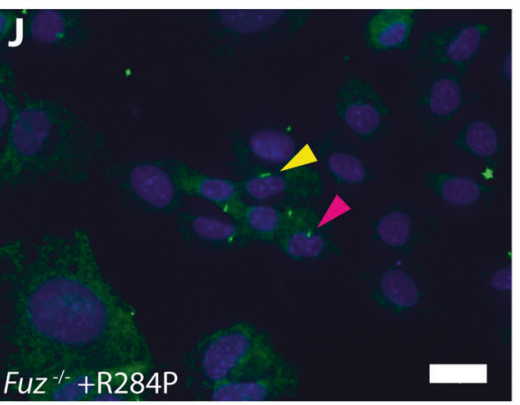

M

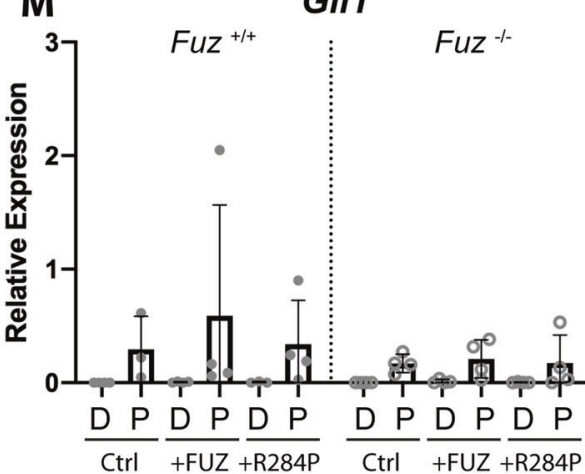

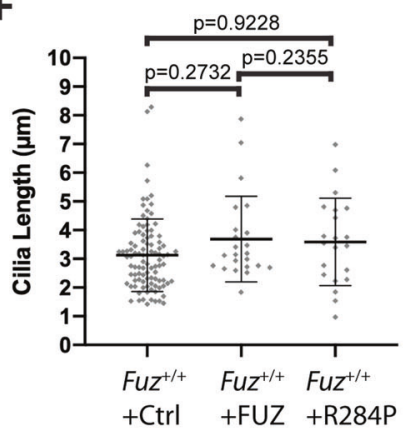

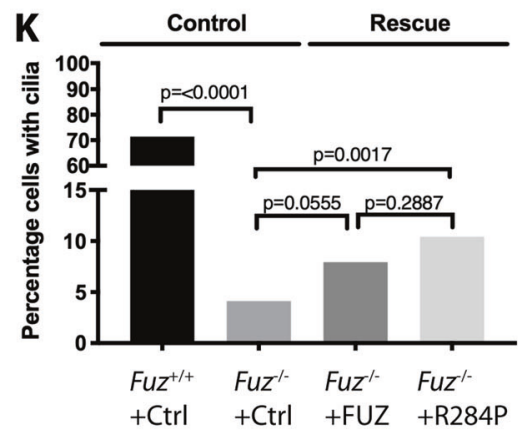

Fig. 3 FUZ p.(Arg284Pro) partially rescues in vitro ciliogenesis phenotype. Both GFP-FUZ-FLAG (+FUZ) or GFP-FUZ p.Arg284Pro-FLAG $(+\mathrm{R} 284 \mathrm{P})$ transfected in immortalised wildtype MEFs show similar cytoplasmic localisation (A-B, anti-GFP (green)). Transfection of GFP-FUZFLAG (+FUZ) or GFP-FUZ p.Arg284Pro-FLAG (+R284P) resulted in no significant change in primary cilium length (measured from anti-antiArl13b staining (magenta), quantified in (F). Rescue experiments were performed in primary MEFs. Ciliary axoneme staining (G-J, anti-Arl13b, green) revealed significantly decreased numbers of cilia present in mutant ( $\mathbf{H}$ and $\mathbf{K}, 3 \%$ of cilia positive cells) compared to control GFP transfected cells ( $\mathbf{G}$ and $\mathbf{K}, 70 \%$ cilia positive cells, $p=<0.0001$, (K). Mutant cells transfected with FUZ (I) or FUZ p.(Arg284Pro) constructs (J) exhibited $4 \%(\mathbf{K}, p=0.0555)$ and $7 \%(\mathbf{K}, p=0.0017)$ increase in cilia number compared to mutant cells transfected with GFP. Cilia were present as dots (G-J, pink arrowhead) or lines (G-J, yellow arrowhead) and were both included in quantification. RT-qPCR relative expression for Hedgehog transcriptional readouts Ptch1 and Gli1 (L and $\mathbf{M}$ ) from immortalised MEFs. Data grouped by genotype as indicated with DMSO [D] or $2 \mu \mathrm{M}$ Purmorphamine [P] treatments and no transfection (Ctrl), transfection of GFP-FUZ-FLAG (+FUZ) or GFP-FUZ p.Arg284Pro-FLAG $(+\mathrm{R} 284 \mathrm{P})$. Statistics and $P$ values $(\mathbf{F})$ are student's $T$ tests and $(\mathrm{K})$ are Fisher's exact tests comparing conditions as indicated. Scale bars $(\mathbf{A}, \mathbf{B})=$ $10 \mu \mathrm{m},(\mathbf{C}-\mathbf{E})=3 \mu \mathrm{m},(\mathbf{G}-\mathbf{J})=50 \mu \mathrm{m}$.

or signalling function of the primary cilium is also impaired. We expressed GFP-fused versions of both variants in wildtype MEFS in order to test their localisation, and found that both forms localised to the cytoplasm (Fig. 3A, B). We then examined their effects on ciliogenesis. Normally, MEFs cultured in reduced serum media will each generate a single primary cilium. Using this assay we checked whether overexpression of FUZ could increase the length of the cilium as previously reported in MDCK cells;[8] however, we did not observe any increase in length, based on staining for the ciliary marker Arl13b (Fig. 3C-F). 
We then used MEFs from Fuz knockout mice to assess whether p.(Arg284Pro) variant could rescue ciliogenesis, due to the observation that MEFs from $\mathrm{Fuz}^{-1-}$ mutant animals very rarely generate cilia ( $3 \%$ (Fig. $3 \mathrm{H}$ and $\mathrm{K}, n=315$, compared to $70 \%$ wildtype MEFs Fig. 3G, K, $n=168$ ). Fuz ${ }^{-1-}$ MEFs were transfected with either a control GFP plasmid, a full-length wild-type FUZ construct, or the variant FUZ-p.(Arg284Pro) construct and assessed for cilia formation. Transfection efficiencies were low; however, the wild-type FUZ construct rescued ciliogenesis in a significant proportion of cells ( $7 \%$ seen in Fig. $3 \mathrm{I}$ and $\mathrm{K}, n=277$, $p=0.0555$ compared to $\mathrm{Fuz}^{-/-}$). Similarly, transfection with the FUZ-p.(Arg284Pro) variant construct increased the proportion of cells with a cilium to $10 \%$ (Fig. $3 \mathrm{~J}, \mathrm{~K}, n=403, p=0.0017$ compared to $\mathrm{Fuz}^{-1-}$ ). Altogether, this suggested that the $\mathrm{p}$. (Arg284Pro) variant could function similarly to the wild-type protein during ciliogenesis.

\section{Inefficient transduction of the Hedgehog signal response by FUZ p.(Arg284Pro)}

While FUZ p.(Arg284Pro) appeared to restore some ciliogenesis, this is relatively unsurprising since the $\mathrm{Fuz}^{-/-}$mice do initially have functional cilia. A defect in Hedgehog signal transduction arises subsequently, likely due to poorly functioning of retrograde IFT leading to cilia degeneration [19]. To address the ciliary function of FUZ p.(Arg284Pro) we compared the ability of wildtype human FUZ and the FUZ p.(Arg284Pro) variant to increase $\mathrm{Hh}$ responsiveness in both control and mutant Fuz MEFs. We performed RT-qPCR for two transcriptional targets of Hedgehog, Patched-1 (Ptc1) and Gli1. Ptc1 is thought to be an immediate early response to $\mathrm{Hh}$ activation, while Gli1 requires a sustained and higher Hh cue. First, we note that the wildtype MEFs respond robustly to treatment with $2 \mu \mathrm{M}$ purmorphamine, which binds to Smoothened and mimics $\mathrm{Hh}$ ligand activation (Figure $\mathrm{L}$ and $\mathrm{M}$, $\left.\mathrm{Fuz}^{+/+}, \mathrm{P}, \mathrm{Ctrl}\right)$. In contrast, Fuz ${ }^{-/-}$MEFs do respond, but less robustly (Fig. $3 \mathrm{~L}, \mathrm{M}$ ). In control MEFs, transfection with wildtype FUZ increases expression of Ptch1 over baseline levels. Transfection of the FUZ p.(Arg284Pro) variant also increases target gene activation, but less than the wild-type (Fig. $3 \mathrm{~L}-\mathrm{M}, \mathrm{Fuz}^{+/+}, \mathrm{P}$, Arg284Pro). The variability in these experiments is likely due to transfection efficiencies; therefore, future experiments should be performed using stable transgenic lines, with further confirmation by immunolocalisation of $\mathrm{Hh}$ pathway effectors. Nevertheless, our observations support the idea that normal MEFs carrying the FUZ p.(Arg284Pro) variant are less efficient when transducing Hedgehog signals. In Fuz ${ }^{-1-}$ MEFS, we found that transfection of either human FUZ variant was sufficient to increase both Ptc1 and Gli1 transcription; however, response to FUZ p.(Arg284Pro) was increased with regards to Ptc1 while we saw almost no response in Gli1.

\section{DISCUSSION}

The genetic aetiology of craniosynostosis is poorly understood. Here, we report a novel craniosynostosis associated mutation in FUZ (c.851 G > C, p.(Arg284Pro)). We found that loss of Fuz resulted in increased mineralisation in both in vitro embryonic primary osteoblast cultures and in fibroblasts undergoing an osteogenic challenge. A direct effect of FUZ mutation in the late stages of bone development and mineralisation has not been reported before. In addition, the novel variant also partially rescued the loss of primary cilia phenotype observed in mutant MEFs [8, 12]. From this we can make the following conclusions: that FUZ is a negative regulator of osteoblast mineralisation and that the $\mathrm{p}$.(Arg284Pro) variant is not a complete loss of function during cilia formation. However, this variant may be inefficient when transducing $\mathrm{Hh}$ signalling.

No previous reports have implicated changes in human FUZ in craniosynostosis. However, variations in FUZ have been found in patients with neural tube defects. Three pathogenic variants were previously investigated (Table 1, case 3-5). The p.(Pro39Ser) variant (case 3, Table 1) exhibited increased cell migration in scratch/wound assays but no effect on ciliogenesis. The opposite was true for the p.(Arg404GIn) variant (case 5, Table 1) where ciliogenesis was impacted but cell migration was not. Finally, the p.(Asp354Tyr) variant exhibited both increased cell migration and defective ciliogenesis (case 4, Table 1). This puts forward the possibility that the C-terminal domain of FUZ (including residues 354 and 404) is necessary during ciliogenesis. We suggest that this functional region excludes the Arg284 residue due to the partial rescue of ciliogenesis seen in our experiments (Fig. 3). In the longer term, it will be of interest to investigate ossification ability of the p.(Pro39Ser) variant in comparison to our p.(Arg284Pro) data.

More recently, a mutation leading to an in-frame frameshift and splice donor site removal was reported in FUZ, leading to an early truncation and likely loss of function [9]. This mutation led to prenatal lethality and presented with a phenotype closely resembling short rib polydactyly syndrome type II (OMIM \#613091). The phenotypes observed were small chest and short limbs with polydactyly, cardiac and kidney defects, and a midline facial cleft, most similar to ciliogenesis variants. As this change likely results in a complete loss of function, (due to the early stop), we would expect both ciliogenesis and ossification phenotypes. In support of this, the phenotypes seen in this patient more closely resembles those found in mouse mutants with a complete loss-offunction [12, 24, 31]. Zhang et al. 2018 [9] also reports a 'unsolved case' variant of FUZ at p.(Arg284Leu). If this mutation is the true cause of this ATD case then this supports strongly the hypothesis that variants at this locus can cause ciliopathic phenotypes.

This work is the first demonstration that FUZ can act as a negative regulator of osteoblast mineralisation. We propose that ciliopathic function may affect the craniofacial skeleton due to a requirement for cilia dependent signalling at several stages during osteogenesis. While a requirement for cilia are well-established during induction of the neural crest precursors of the cranial skeleton, the later requirements during bone formation and mineralisation are less appreciated. The reported gene variants of FUZ including the novel p.(Arg284Pro) variant provide us with an opportunity to dissect the sequential uses of FUZ and CPLANE proteins during development of the craniofacial skeleton. In the case of FUZ, these variants could be introduced to primary cells or cell lines using CRISPR, or virally into mutant cells, and subsequently used to test specific functions, which in the long term could separate the functional domains controlling vesicle trafficking, osteoblast mineralisation and ciliogenesis functions of FUZ.

Based on animal models and human case studies, it is clear that genetic mutations affecting the structure and function of the primary cilium can result in developmental abnormalities and skeletal dysplasias [33]. These often affect the limbs with polydactyly and the face with cleft lip/palate, micrognathia, facial width abnormalities and craniosynostosis. Several ciliopathies that predominantly affect the craniofacial skeleton present with craniosynostosis [22]. Together, this suggests an overlap between ciliopathic syndromes and craniosynostosis $[29,30]$ and attributes a subset of these to Hedgehog signalling changes.

Hh signalling can affect both osteoblast differentiation and mineralisation. Indian Hedgehog $(\mathrm{IHH})$ signalling is necessary for the early differentiation of osteoblasts, with upregulation of $\mathrm{Hh}$ signalling via the small molecule purmorphamine resulting in increased commitment to osteoblastic maturation [34]. A loss at this stage could leave osteoprogenitors in a proliferative and undifferentiated state, effectively increasing the number of precursors able to differentiate and mineralise. In contrast, loss of Hh signalling, via a conditional knockout of the Smoothened receptor in mature osteoblasts, results in increased bone mass and a disruption in bone homeostasis [35]. Thus, it is evident that $\mathrm{Hh}$ 
signalling is necessary at several key time points during skeletal development and that loss of FUZ may reduce the Hh signalling capacity via the primary cilium at these critical stages.

However, simple non-syndromic craniosynostoses are often surgically treated and whether there is a genetic aetiology is not assessed. These cases could be caused by partial loss of function variants in multifunction genes, like FUZ. Where complete loss of function would cause systemic anomalies; here, a missense or partial loss of function mutation may affect a specific functional region of the protein giving a 'microform' presentation of a syndrome. Linking genetic variants to the functional regions of proteins will help us understand the phenotypic manifestation of the syndrome, as well as the relative severity. This will help with genetic counseling, diagnosis and may determine what future follow-up is required.

\section{MATERIALS AND METHODS \\ Sequencing}

Whole genome sequencing (WGS) was performed on DNA from blood by Complete Genomics, (Mountain View, CA, USA) [36]. Variants were annotated using NCBI build GRCh37/hg19 and dbSNP build 137. Data were analyzed using cga tools version 1.8.0. An autosomal recessive disease model was tested. The analysis was restricted to novel non-synonymous variants, variants disrupting a splice site ( \pm two basepairs), and insertions or deletions in the coding sequence $( \pm 50 \mathrm{bp})$. The remaining variants were analyzed with Annovar [37] and OpenCravat [38] to get an indication of the pathogenicity and allele frequency, and compared to those present in the wellderly cohort [39]. The variant identified was described according to HGVS nomenclature [40], using reference sequence NM_025129.5, on GRCh37/hg19 and was submitted to the Leiden Open Variation Database. The variant identified by WGS was validated by dideoxy-sequence analysis.

\section{Mouse lines and animal husbandry}

Fuz mutants (MGl:3531090) in this study were previously reported [12]. All animal work was carried out in accordance with UK Home Office regulations under the project licence P8D5E2773 held by KJL. Immortalised MEFs were derived from another previously reported Fuz mutant line [8].

\section{Cell culture}

Primary mouse embryonic fibroblasts (MEFs) were isolated from E12.5 embryos using standard procedures (Fig. 2, Fig. 3 G-K), while immortalised MEFs have been previously described (used in Fig. 3 A-E and L, M) [8]. MEFs were passaged every 3-4 days and primary MEFs were used up to passage 5. DNA transfections were performed with Lipofectamine LTX or 2000 reagent (Thermofisher) per manufacturer's protocol. For cilia induction FBS content of MEF media was reduced to $0.5 \%$ for 48 hours.

Primary osteoblasts were isolated from the dissected calvaria and mandibles of staged E18.5 mouse embryos. Cells were plated in a 24-well plate in primary osteoblast growth media (a-MEM (Lonza), 10\% batch tested osteogenic FBS, 1X ABAM, 1X L-glutamine) and incubated at $37^{\circ} \mathrm{C}$ with $5 \% \mathrm{CO}_{2}$. When confluent, cells were trypsinised and chips allowed to sediment. The resulting cell solution was then passed through a 40um cell strainer. Cells were resuspended to 40,000 cells/well, plated into a 48 well plate and cultured for 10 days in osteoblastic mineralisation medium (primary osteoblast growth media, $50 \mathrm{ug} / \mathrm{ml}$ ascorbic acid, $5 \mathrm{mM} \beta$ glycerophosphate). For MEF to osteoblast differentiation primary MEFs were cultured in osteoblastic mineralisation media $+50 \mathrm{ng} / \mathrm{ml}$ BMP2. Standard protocols for alkaline phosphatase staining and subsequently Von Kossa staining were then carried out.

\section{Reverse transcription-quantitative PCR}

RNA was extracted using Trizol (Sigma), followed by cDNA synthesis according to conventional methods [41]. The following quantitative PCR primers were used: mouse $\beta$-actin: for-CTAAGGCCAACCGTGAAAAG3', revACCAGAGGCATACAGGGACA; mouse Patched-1 (Ptch1) for-AAGCCGACTAC ATGCCAGAG, rev-AAGGGAACTGAGCGTACTCG; mouse Gli1 for- CAGGGAA GAGAGCAGACTGAC, rev CGCTGCTGCAAGAGGACT.

\section{Immunofluorescent antibody staining}

Primary MEFs were fixed in $100 \%$ Methanol for 15 mins on ice. After washing ( $1 \%$ BSA, $0.1 \%$ Tween 20 in $1 \mathrm{X}$ PBS) cells were permeabilised with
$0.5 \%$ TritonX-100 (in 1X PBS) and blocked in (3\% BSA, 10\% Goat serum, $0.1 \%$ Tween 20, in 1X PBS) for 1 hour at room temperature. Anti-ARL13b primary antibody (1:500, Proteintech, 17711-1-AP) was incubated in blocking buffer overnight at $4{ }^{\circ} \mathrm{C}$.

Immortalised MEFs were fixed in 4\% PFA for 10 mins at room temperature and stained with anti-GFP (1:500, Abcam, ab13970) and anti-Arl13b (as before) for 1 hour at room temperature. Coverslips were then washed and incubated in anti-rabbit 488, anti-Chicken 488 or anti-Rabbit 568 secondary (1:500, Invitrogen, A11008, A11039 and A11011) for 2 hours at room temperature. Nuclei were stained with Hoechst 33342 (Sigma) 1:1000 (20 $\mathrm{mg} / \mathrm{ml}$ stock). Plates were imaged using standard inverted epifluorescent microscope (Zeiss) or confocal (Nikon A1R). Image processing was carried out in FIJI (ImageJ) [42]. Cilia length was measured using maximum intensity projections from Arl13b staining in GFP positive cells (indicating transfection).

\section{Protein predictions and secondary structure prediction}

STRING queries (https://string-db.org/) were rooted on human FUZ protein identifier ENSP00000313309 showing functional and physical protein associations. Human FUZ amino acid sequence was analysed using PHYRE2 (an ab initio and homology modelling tool) [43]

\section{DATA AVAILABILITY}

Data available within the article or upon reasonable request. The p.(Arg284Pro) variant is reported in the Leiden Open Variation Database (https://databases.lovd.nl/ shared/individuals/00375537).

\section{REFERENCES}

1. Johnson D, Wilkie AOM. Craniosynostosis. Eur J Hum Genet. 2011;19:369-76.

2. Lajeunie E, Le Merrer M, Bonaiti-Pellie C, Marchac D, Renier D. Genetic study of nonsyndromic coronal craniosynostosis. Am J Med Genet. 1995;55:500-4.

3. Cornelissen $M$, Ottelander $B$ den, Rizopoulos $D$, van der Hulst R, Mink van der Molen A, van der Horst $C$, et al. Increase of prevalence of craniosynostosis. J Cranio-Maxillofac Surg. 2016;44:1273-9.

4. Wilkie AOM, Johnson D, Wall SA. Clinical genetics of craniosynostosis. Curr Opin Pediatr 2017;29:622-8.

5. Beederman M, Farina EM, Reid RR. Molecular basis of cranial suture biology and disease: osteoblastic and osteoclastic perspectives. Genes Dis. 2014;1:120-5.

6. Hall B. Bones and cartilage: developmental and evolutionary skeletal biology. Second Ed. San Diego, California: Elsevier Academic Press; 2015.

7. Heuzé Y, Holmes G, Peter I, Richtsmeier JT, Jabs EW. Closing the gap: genetic and genomic continuum from syndromic to nonsyndromic craniosynostoses HHS public access. Curr Genet Med Rep. 2014;1:135-45. Sept

8. Seo JH, Zilber Y, Babayeva S, Liu J, Kyriakopoulos P, De Marco P, et al. Mutations in the planar cell polarity gene, Fuzzy, are associated with neural tube defects in humans. Hum Mol Genet. 2011;20:4324-33.

9. Zhang W, Taylor SP, Ennis HA, Forlenza KN, Duran I, Li B, et al. Expanding the genetic architecture and phenotypic spectrum in the skeletal ciliopathies. Hum Mutat. 2018;39:152-66.

10. Rossi V, Banfield DK, Vacca M, Dietrich LEP, Ungermann C, D'Esposito $M$, et al. Longins and their longin domains: regulated SNAREs and multifunctional SNARE regulators. Trends Biochem Sci. 2004;29:682-8.

11. Daste F, Galli T, Tareste D. Structure and function of longin SNAREs. J Cell Sci. 2015;128:4263-72.

12. Gray RS, Abitua PB, Wlodarczyk BJ, Szabo-Rogers HL, Blanchard O, Lee I, et al. The planar cell polarity effector Fuz is essential for targeted membrane trafficking, ciliogenesis and mouse embryonic development. Nat Cell Biol. 2009;11:1225-32.

13. Toriyama M, Lee C, Taylor SP, Duran I, Cohn DH, Bruel A-L, et al. The ciliopathyassociated CPLANE proteins direct basal body recruitment of intraflagellar transport machinery. Nat Genet. 2016;48:648-56.

14. Sanchez-Pulido L, Ponting CP. Hexa-Longin domain scaffolds for inter-Rab signalling. Bioinformatics. 2019. https://doi.org/10.1093/bioinformatics/btz739.

15. Gerondopoulos A, Strutt H, Stevenson NL, Stephens DJ, Strutt D, Correspondence FAB planar cell polarity effector proteins inturned and fuzzy form a Rab23 GEF complex in brief. Curr Biol. 2019; 29. https://doi.org/10.1016/j.cub.2019.07.090.

16. Wong LL, Adler PN. Tissue polarity genes of Drosophila regulate the subcellular location for prehair initiation in pupal wing cells. J Cell Biol. 1993;123:209-21.

17. Collier S, Gubb D. Drosophila tissue polarity requires the cell-autonomous activity of the fuzzy gene, which encodes a novel transmembrane protein. Development. 1997;124:4029-37.

18. Park TJ, Haigo SL, Wallingford JB. Ciliogenesis defects in embryos lacking inturned or fuzzy function are associated with failure of planar cell polarity and Hedgehog signaling. Nat Genet. 2006;38:303-11. 
19. Brooks ER, Wallingford JB. Control of vertebrate intraflagellar transport by the planar cell polarity effector Fuz. J Cell Biol. 2012;198:37-45.

20. Ishikawa H, Marshall WF. Ciliogenesis: building the cell's antenna. Nat Rev Mol Cell Biol. 2011;12:222-34.

21. Bangs F, Anderson KV. Primary cilia and Mammalian Hedgehog signaling. Cold Spring Harb Perspect Biol. 2017;9:1-22.

22. Schock EN, Brugmann SA Discovery, Diagnosis, and Etiology of Craniofacial Ciliopathies. Cold Spring Harb Perspect Biol. 2017; 9. https://doi.org/10.1101/ cshperspect.a028258.

23. Brugmann SA, Cordero DR, Helms JA. Craniofacial ciliopathies: a new classification for craniofacial disorders. Am J Med Genet 2010;152 A:2995-3006. Part A.

24. Yannakoudakis BZ, Liu KJ. Common skeletal features in rare diseases: New links between ciliopathies and FGF-related syndromes. Rare Dis (Austin, Tex). 2013;1: e27109.

25. Lenton K, James AW, Manu A, Brugmann SA, Birker D, Nelson ER, et al. Indian hedgehog positively regulates calvarial ossification and modulates bone morphogenetic protein signaling. Genesis. 2011;49:784-96.

26. St-Jacques B, Hammerschmidt M, McMahon AP. Indian hedgehog signaling regulates proliferation and differentiation of chondrocytes and is essential for bone formation. Genes Dev. 1999;13:2072-86.

27. Yang J, Andre $P, Y e L$, Yang Y-Z. The Hedgehog signalling pathway in bone formation. Int J Oral Sci. 2015;7:73-9.

28. Agbu SO, Liang Y, Liu A, Anderson KV The small GTPase RSG1 controls a final step in primary cilia initiation. J Cell Biol 2017; 217: jcb.201604048.

29. Tabler J, Barrell W, Szabo-Rogers H, Healy C, Yeung Y, Perdiguero E, et al. Fuz Mutant Mice Reveal Shared Mechanisms between Ciliopathies and FGF-Related Syndromes. Dev Cell. 2013;25:623-35.

30. Tabler JM, Rice CP, Liu KJ, Wallingford JB. A novel ciliopathic skull defect arising from excess neural crest. Dev Biol. 2016;417:4-10.

31. Zhang Z, Wlodarczyk BJ, Niederreither K, Venugopalan S, Florez S, Finnell RH et al. Fuz regulates craniofacial development through tissue specific responses to signaling factors. PLoS One 2011; 6. https://doi.org/10.1371/journal.pone.0024608.

32. Adel Al-Lami H, Barrell WB, Liu KJ. Micrognathia in mouse models of ciliopathies. Biochem Soc Trans. 2016;44:1753-9.

33. Haycraft CJ, Serra R. Cilia involvement in patterning and maintenance of the skeleton. Curr Top Dev Biol. 2008;85:303-32.

34. Wu X, Ding S, Ding Q, Gray NS, Schultz PG. A small molecule with osteogenesisinducing activity in multipotent mesenchymal progenitor cells. J Am Chem Soc. 2002;124:14520-1.

35. Mak KK, Bi Y, Wan C, Chuang PT, Clemens T, Young M, et al. Hedgehog Signaling in Mature Osteoblasts Regulates Bone Formation and Resorption by Controlling PTHrP and RANKL Expression. Dev Cell. 2008;14:674-88.

36. Drmanac R, Sparks AB, Callow MJ, Halpern AL, Burns NL, Kermani BG, et al. Human genome sequencing using unchained base reads on self-assembling DNA nanoarrays. Science (80-). 2010;327:78-81.

37. Wang K, Li M, Hakonarson H ANNOVAR: Functional annotation of genetic variants from high-throughput sequencing data. Nucleic Acids Res 2010; 38. https://doi. org/10.1093/nar/gkq603.

38. Pagel KA, Kim R, Moad K, Busby B, Zheng L, Tokheim C, et al. Integrated Informatics Analysis of Cancer-Related Variants. JCO Clin Cancer Inform. 2020;4:310-7.

39. Erikson GA, Bodian DL, Rueda M, Molparia B, Scott ER, Scott-Van Zeeland AA, et al. Whole-Genome Sequencing of a Healthy Aging Cohort. Cell. 2016;165:1002-11.

40. Den Dunnen JT, Antonarakis SE. Mutation nomenclature extensions and suggestions to describe complex mutations: A discussion. Hum Mutat. 2000;15:7-12.

41. Barrell WB, Griffin JN, Harvey J-L, Danovi $D$, Beales $P$, Grigoriadis $A E$, et al. Induction of Neural Crest Stem Cells From Bardet-Biedl Syndrome Patient Derived hiPSCs. Front Mol Neurosci. 2019;12:139.

42. Schindelin J, Arganda-Carreras I, Frise E, Kaynig V, Longair M, Pietzsch T, et al. Fiji: An open-source platform for biological-image analysis. Nat Methods 2012;9:676-82.

43. Kelley LA, Mezulis S, Yates CM, Wass MN, Sternberg MJE. The Phyre2 web portal for protein modeling, prediction and analysis. Nat Protoc. 2015;10:845-58.

\section{ACKNOWLEDGEMENTS}

We thank members of the Liu lab as well as Agi Grigoriadis for discussions. We especially thank Emily Lodge for critical reading of the manuscript. We also thank the Centre for Craniofacial and Regenerative Biology and the King's College London Biological Services Unit for their support.

\section{AUTHOR CONTRIBUTIONS}

JACG, SMAS, MvD, PJvdS, IMJM contributed to clinical evaluation and sequencing analysis. WBB, HAA and KJL designed and performed experiments. ET provided cell lines. All authors contributed to the writing of the manuscript.

\section{FUNDING}

This study was funded by grants from the EPSRC (WBB/KJL), BBSRC BB/R015953/1 (KJL) and studentship from the Iraq Higher Council for Education (HAA). Bioinformatics data analysis and whole genome sequencing infrastructure has been funded from grants at Erasmus medical including CTMM Biochip, CTMM Trait, ZonMW/NWO Genetics First, H2020 Bigmedilytics and the Erasmus Center for Data Analytics (ECDA).

\section{ETHICAL APPROVAL}

The clinical study was approved by the Medical Ethical Committee of the Erasmus University Medical Center Rotterdam (MEC-2012-140). Written informed consent to obtain samples for genetics research was given by the parents.

\section{COMPETING INTERESTS}

The authors declare no competing interests.

\section{ADDITIONAL INFORMATION}

Supplementary information The online version contains supplementary material available at https://doi.org/10.1038/s41431-021-00988-6.

Correspondence and requests for materials should be addressed to Karen J. Liu.

Reprints and permission information is available at http://www.nature.com/ reprints

Publisher's note Springer Nature remains neutral with regard to jurisdictional claims in published maps and institutional affiliations.

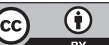

Open Access This article is licensed under a Creative Commons Attribution 4.0 International License, which permits use, sharing, adaptation, distribution and reproduction in any medium or format, as long as you give appropriate credit to the original author(s) and the source, provide a link to the Creative Commons license, and indicate if changes were made. The images or other third party material in this article are included in the article's Creative Commons license, unless indicated otherwise in a credit line to the material. If material is not included in the article's Creative Commons license and your intended use is not permitted by statutory regulation or exceeds the permitted use, you will need to obtain permission directly from the copyright holder. To view a copy of this license, visit http://creativecommons. org/licenses/by/4.0/.

(c) The Author(s) 2021 